\title{
Digital Technology in Diagnostic Breast Pathology and Immunohistochemistry
}

\author{
Emad A. Rakha ${ }^{a}$ Konstantinos Vougas ${ }^{b, c}$ Puay Hoon Tan ${ }^{d}$ \\ aDepartment of Histopathology, School of Medicine, The University of Nottingham and Nottingham University \\ Hospitals NHS Trust, Nottingham City Hospital, Nottingham, UK; ${ }^{b}$ Biomedical Research Foundation of the Academy \\ of Athens, Athens, Greece; 'DeepMed IO Ltd, Manchester, UK; ${ }^{d}$ Division of Pathology, Singapore General Hospital, \\ Singapore, Singapore
}

\section{Keywords}

Digital pathology $\cdot$ Immunohistochemistry $\cdot$ Artificial intelligence $\cdot$ Whole slide image $\cdot$ Diagnosis $\cdot$ Applications

\begin{abstract}
Digital technology has been used in the field of diagnostic breast pathology and immunohistochemistry (IHC) for decades. Examples include automated tissue processing and staining, digital data processing, storing and management, voice recognition systems, and digital technology-based production of antibodies and other IHC reagents. However, the recent application of whole slide imaging technology and artificial intelligence (Al)-based tools has attracted a lot of attention. The use of Al tools in breast pathology is discussed briefly as it is covered in other reviews. Here, we present the main application of digital technology in IHC. This includes automation of IHC staining, using image analysis systems and computer vision technology to interpret IHC staining, and the use of Al-based tools to predict marker expression from haematoxylin and eosin-stained digitalized images.

(c) 2021 S. Karger AG, Basel
\end{abstract}

\section{Introduction}

Digital technology is an umbrella term for computerbased products and solutions, and it includes electronic systems, tools, and devices that generate, store, or process data. Digital technology tools have been used in pathology for many years and examples include computers, storage devices, digital microscope cameras, dictation machines, voice recognition systems, digital automated tissue processors and autostainers, computerized generation of synoptic reports, and recently digital whole slide image (WSI) technology and artificial intelligence (AI)based algorithms for image processing. Digital technology has been progressing faster than any invention in human history and has transformed and revolutionized many aspects of our lives including pathology. Digital technology often means that huge amounts of information can be stored, processed, and moved almost instantaneously, and that devices have generally become much faster, smaller, and more versatile. The growth of electronic health records, the advancement in computational approaches for analysing "big data," and pathology image processing with progress in computer vision technology have created an opportunity to establish a new discipline within pathology, computational pathology (CPATH), 
which encompasses digital pathology (DP) and machine learning (ML)/AI-based approaches.

The term CPATH can be defined as the "omics" or "big data" approach to pathology, where multiple sources of patient information including pathology image data and meta-data are combined to extract patterns and analyse features [1]. CPATH is currently a branch of pathology that involves computational analysis of a broad array of methods to analyse pathology samples for the study of disease. With the future expansion and use of AI tools, CPATH is expected to incorporate multiple sources of data in addition to pathology (e.g., radiology, clinical, and molecular) and use more advanced mathematical models to generate a comprehensive diagnostic and prognostic outcome to guide treatment decision-making. CPATH is expected to maintain and improve the efficiency of pathology and laboratory services in this era of precision medicine, with increasingly complex requirements of cellular pathology and the continuous need to improve patient healthcare. The term computer vision refers to a scientific field that deals with how computers can be made to gain high-level understanding from digital images, including methods for acquiring, processing, analysing, and understanding digital images and extraction of data to produce numerical or symbolic information [1]. Computer vision and AI share other methods such as pattern recognition and learning techniques.

Despite the overlap in the terminology and the use of the terms interchangeably, in this article, these terms are defined as follows. Conventional image analysis refers to the extraction of meaningful information such as finding shapes, counting objects, identifying colours, or measuring object properties from digital images employing digital image processing techniques. The aforementioned information extraction process is performed by a human expert, and the result is a number of "handcrafted" features. Those features are used as input either in statistical analysis for pattern recognition and knowledge extraction or in traditional ML frameworks for classification and segmentation. On the other hand, the application of AI on WSIs, as implied in the current review, is based on deep learning neural networks, which operate directly on the raw image pixels with specific goals (e.g., classification, object detection, or segmentation) and construct features internally and autonomously as part of the learning process, in contrast to the aforementioned "handcrafted" features. In this case, knowledge extraction is performed retrospectively by AI-explainability methodologies aiming to translate internal network dynamics into human interpretable information. In other words, deep learning is a subfield of ML, and ML is a subfield of AI. That means an AI-based WSI analysis pipeline can be created by ML or deep learning models to accomplish tasks such as image classification and segmentation. However, the main difference between ML and deep learning is that in an ML model or the conventional image analyses, we need to find/extract the set of features from the images manually, which is a laborious and hard process to train the model, while in deep learning, here refers to AI, the model can learn the features itself.

The recent introduction of WSI technology in routine pathology practice, considered as a paradigm shift technology with the ability to integrate ML/AI-based algorithms, computer vision, and image processing tools into the routine pathology workflow, has attracted a lot of attention. Subsequently, it opened avenues for more investment in this rapidly expanding field with myriad potential applications of ML/AI technology. WSI technology is the process of transforming histopathology slides into digital images using whole-slide scanners and subsequent viewing, and possibly analysis, of these digitized images using image management and viewing software. The terms DP and CPATH are becoming commonplace. The use of computer vision coupled with deep learning approaches to determine specific features of WSI can provide information far beyond the ability of pathologists and enable the utilization and integration of knowledge beyond human limits and boundaries and in a very short time. By using WSI and CPATH, many tasks in pathology departments can become more automated, standardized, and cost-efficient.

Immunohistochemistry (IHC) is increasingly used in pathology as a diagnostic, prognostic, and predictive tool not only to detect the existence of molecules but also to identify their spatial relationships. IHC, which dates back decades, is not stagnant but evolving fast. Automated sample processors, digital technology-based antibody and reagent development, digital slide scanners, image analysis software, computer vision, and more are changing the dynamics of IHC, and we expect the second-generation and third-generation IHC to come to clinical practice soon.

In this article, we explore CPATH in diagnostic breast pathology and IHC. We start with an overview of DP, a prerequisite for the application of ML/AI-based tools, and the use of AI applications in diagnostic breast pathology. Then, we discuss the details of the use of CPATH in IHC. This includes the application of DP in the pre-analytical, analytical, and post-analytical phases and the use of $\mathrm{AI}$ and image analysis tools for interpretation of IHC 


\section{Applications of digital technology (DT) in breast pathology}

\begin{tabular}{|c|}
\hline Workflow \\
\hline $\begin{array}{l}\text { Improving efficiency of workflow } \\
\text { and streamline the process }\end{array}$ \\
\hline $\begin{array}{l}\text { Digitalisation of tissue processing } \\
\text { and staining }\end{array}$ \\
\hline Automatic QC of slides \\
\hline Automatic requests of extra-tests \\
\hline $\begin{array}{l}\text { Digital dictation and automated } \\
\text { reporting }\end{array}$ \\
\hline
\end{tabular}

Diagnostic

\begin{tabular}{|c|}
\hline Remote reporting \\
\hline Tumour detection algorithms \\
\hline $\begin{array}{l}\text { Scoring variables quantitatively } \\
\text { such as TILs, and mitotic counts }\end{array}$ \\
\hline
\end{tabular}

Providing primary reporting or secondary reporting of subjective cases (double reporting)

Automatic auditing of cases
Prognostic and predictive

Correlate myriads of morphological features with molecular profiles, behaviour and response to therapy to provide prognostic and predictive assays and novel markers

Integration of pathology reports with imaging and omics data to provide comprehensive report for accurate patients managment.

Automated follow up of patients

\section{Other applications of DT in pathology}

Helps pathologists by providing lists of differential diagnosis, virtual bank of images of difficult and rare cases, and suggests markers and extra-tests in challenging cases. Also, it allows merging of published national guidelines

a

Applications of digital technology (DT) in immunohistochemistry (IHC)

\begin{tabular}{|l|}
\hline \multicolumn{1}{|c|}{ Preanalytical } \\
\hline $\begin{array}{l}\text { Development and manufacture } \\
\text { of antibodies and detecion } \\
\text { systems }\end{array}$ \\
\hline
\end{tabular}

Search and characterisation of antibodies and reagents (QC and QA of staining)

Improving processing of tissues

Automated requests of $\mathrm{IHC}$ stains

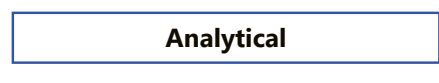

Automation of staining

Improving workflow efficieny

Multiplexing of IHC

Automatic QC of staining

Scanning of stained slides

Digitalisation of stained slides

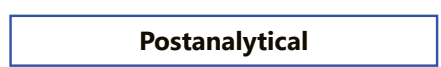

Use of image analysis and computer vision tools

Automation of scoring

High throughput analysis of marker expression heterogeneity and spatial distribution

Integration of IHC results with other relevant variables to improve interpretation

\section{Other applications of DT in IHC}

Automated reporting systems, virtual IHC technology and centralisation of the staining process with the use of DP b

Fig. 1. Applications of digital technology in breast pathology (a) and IHC (b). DT, digital technology. 
markers including the diagnostic and prognostic and predictive markers. Finally, we address outstanding challenges in the field and promising future directions.

\section{Applications of CPATH in Breast Pathology}

There are several applications of CPATH in breast pathology including general and breast pathology-related applications (Fig. 1a). General applications include (i) improving the pathology workflow efficiency by reducing reporting turnaround time and streamlining the process of specimen workflow and routine diagnostic pathways, (ii) automation and reducing error rates, (iii) specimen and report quality control and quality assurance measures, (iv) audits, training, and validation processes, (v) slide sharing, and (vi) facilitating junior doctor and biomedical scientist reporting in addition to (vii) storage benefits. It can also improve the quality of the pathology service by (viii) strengthening communications with stakeholders and service users, (ix) increasing the ability to link multiple test results, and producing comprehensive reports that contain multi-parameter data input.

In breast pathology, specific roles include (i) diagnostic applications and (ii) providing cost-efficient and novel prognostic and predictive assays in addition to (iii) automation of some processes related to routine breast pathology practice such as automatic requests of receptors in malignant cases and scoring some features such as TILs. The application of AI tools is expanding at a high speed not only to improve current practice by helping pathologists report routine cases or improving service efficiency but also provide information far beyond the current ability. AI algorithms to predict the genomic and protein profiles of tissues from the morphology of $\mathrm{H} \& \mathrm{E}$ stained WSI and extract myriad information from these slides to provide novel molecular and morphological diagnostic, prognostic, and predictive tools can replace or even outperform existing tools [2-4]. Interestingly, evidence has been provided that CPATH can even effectively replace established molecular tests, measuring the gene-expression level of specific biomarker panels for the prediction of long-term recurrence and chemotherapy benefits such as Oncotype DX. For instance, Klimov et al. [5] demonstrated that it is possible for ML models to accurately predict the probability of recurrence in breast cancer patients with ductal carcinoma in situ (DCIS). Specifically, the group trained an ML pipeline on a retrospective cohort of 159 DCIS patients and predicted the 10 -year recurrence probability for a blind retrospective

Digital Technology and IHC in Breast Pathology cohort of 185 DCIS patients, achieving an accuracy of $85 \%$. Details about the application of CPATH in breast pathology were addressed in detail in other reviews [613]. In this review, we focus on the application of CPATH in IHC of the breast including diagnostic and predictive IHC markers. The applications include not only improving the performance of IHC in the pre-analytical, analytical, and post-analytical phases but also the recent development in the prediction of IHC expression of biomarkers from the morphology of the tissue and the ability for multiplexing the process.

\section{Immunohistochemistry in Breast Pathology}

In breast pathology, IHC is used for diagnostic purposes, for prognostic stratification of malignant lesions, and as a companion diagnostic for specific therapy (therapeutic purposes). In IHC, antibodies against the epitope(s) of a specific target (also referred to as a "marker," typically a protein but may also be carbohydrates or lipids) are used. IHC detects the presence of a marker(s) and pattern of expression in situ using antigen-antibody reactions and is applied to formalin-fixed paraffin-embedded tissue samples as well as to cytological preparations and fresh frozen samples. Although quantifying IHC staining patterns of diagnostic, prognostic, and predictive markers is of crucial importance, there are pitfalls and staining quality issues related to various markers.

Pathologists refine IHC tests by testing new antibody clones, platforms, and detection reagents and create new IHC tests based on markers discovered through research, diagnostic surveys, or to recapitulate other tests including mutation-specific IHC. Because IHC tests are interpreted visually by a pathologist, marker choice and assay optimization may be regarded as part science, part art.

Guideline recommendations on a limited set of markers such as ER, PR, and HER2 are published $[14,15]$; however, guidelines on the staining and the interpretation of most other markers are lacking, and pathologists rely on published articles and textbooks. The problem is that each of these steps of IHC can be tweaked, and there is no "standard" IHC protocol for all IHC markers that can be used universally. Data on antibodies, IHC staining protocol, interpretation, and scoring are not consistent, and variations exist. The specificity, sensitivity, staining patterns, validation process, antibody clones, detection kits, cross-reactivity, controls and staining pitfalls of each IHC marker can be variable and depend on multiple factors (see pre-analytical issue below). A great deal of variation 
in the performance of IHC can be seen between different tissue types, fixation qualities, IHC staining kit and reagent used, staining condition, and between different observers who may use different thresholds and subjective interpretation. Even in a given laboratory with a wellcontrolled tissue processing and staining workflow, IHCstained slides may be subject to significant variations not only inter-batch staining variations but also with the same batches.

In the clinical laboratory, the gold standard for the evaluation and quantitation of IHC remains manual annotation, which is expensive and may lead to subjective errors. However, CPATH with digital image analysis and AI-based methods is steadily growing and holds much promise for efficient and accurate pattern recognition and quantitation of IHC [16]. Breast cancer IHC biomarkers, including oestrogen receptor, progesterone receptor, HER2, and Ki-67, were the first biomarkers to be analysed using quantitative image analysis. CPATH tools including $\mathrm{AI}$ algorithms, computer vision, and automated image analysis tools have several advantages. CPATH tools can not only overcome the limitations and subjectivity of the pathologists but also provide high-throughput analysis, automation, and multiplexing of IHC far beyond what humans can do without such tools (Fig. 1b).

\section{CPATH and Pre-Analytical and Analytical Phases of IHC}

CPATH can help in improving the pre-analytical factors that can affect the performance of IHC. Not only automation and standardization of the various processes related to tissue handling, fixation and staining in the laboratory especially in those using DP, and WSI technology workflow but also using AI-based quality assurance and QC measures can improve the quality of IHC. Using AI algorithms to improve communication between operating theatres and the pathology laboratory, provide additional guidance of tissue fixation and handling, and manage the sample handling pathways and flag errors in the systems can ensure that all samples are treated according to the approved protocols. Using automation by employing systems that treat slides precisely the same including sample processing and IHC staining systems will reduce variation. One use of CPATH to improve efficiency is the application of AI algorithms to screen slides, and pre-order IHC and some of the commercial AI algorithms have been CE marked for this use, which provide an example of how CPATH/AI is improving workflow at a practical level. AI is currently used in online search engines to help pathologists and lab managers to choose the best kits and reagents to suit the tissue type and local protocols and resources in the laboratory. Digital technology is also employed in the development of IHC reagents including synthesized primary antibodies and detection systems [17].

The continuous increase in computational power, with big data handling and analysis together with substantial advances in the computer vision, digital image processing, and analysis and pattern recognition fields, has motivated the development of computer-aided diagnostic systems and enabled the utilization of all available data on the performance of individual markers that can be implemented in routine practice. This has also attracted more attention given the increasing number of tissue biomarkers and the complexity of their evaluations. The implementation of such systems is likely to change the workflow and refine IHC-related processes including tissue processing and staining to suit the specific markers and local resources and infrastructure. There are commercially available tools that can automate a wide range of tests involving tissue incubation protocols for immunostaining and multiplexing, with the possibility to modify protocol steps, select different incubation times, or choose different reagents to fine tune the immunostaining tests ([18]https://epredia.com/labsat/).

Currently, IHC used in clinical diagnosis mostly interrogates a single marker per tissue section and is unable to characterize cell phenotypes defined by the co-expression of multiple markers. Simultaneous IHC staining reactions can generate 2 or a maximum of 3 markers mainly of different sub-cellular localization and different colour chromogens that can be interpreted using eyeballing assessment [19]. Perhaps one of the most important developments in the analytical phase of IHC is the development of multiplex IHC (mIHC) using novel staining and analysis techniques $[20,21]$. CPATH and $\mathrm{mIHC}$ can form the foundation for the next-generation IHC in pathology $[22,23]$. mIHC permits the labelling of multiple molecules within a single histologic tissue section, and the identification of each molecule requires detection of the unique coloured chromogens localized to the corresponding antigen on the cells expressing the markers of interest (Fig. 2). Currently, multiplexed staining assays using 4 or more markers have been developed [19], permitting direct observation of different cell populations within a specimen, the so-called digital phenotyping, and allowing the evaluation of the cellular spatial relationships. mIHC employs an advancement in the way chromogens work, permitting the development of chromogen stains with narrow and well-separated absorbance bands, 


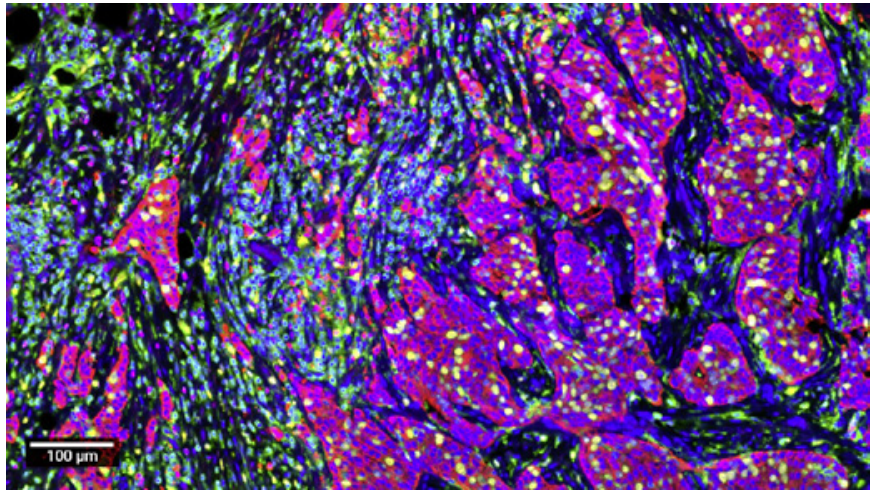

Fig. 2. A triple-negative breast cancer stained using multiplex immunohistochemistry/immunofluorescence (mIHC/IF) (DAPI [blue], CD45 [green], Ki-67 [yellow], CD8 [purple], and EPCAM + Pan-Cytokeratin [red]). Image kindly provided by Dr. Joe Yeong at SGH.

similar to fluorophores [24] but also utilizes special cameras with filters and software that are capable of deciphering the absorbance bands of the discrete chromogens for high-speed multiplex imaging. Other tools such as the CODEX (CO-Detection by indEXing) technology use antibodies conjugated to a proprietary library of oligonucleotides called barcodes which enables customizable panels of more than 40 CODEX antibodies to be combined for a single tissue staining reaction [25]. Recently, a fully automated and ultra-fast multi-staining using a microfluidic tissue processor has been developed, allowing not only multiplexing of staining in a precise and reproducible manner but also with a turnaround time shorter than existing monoplex staining (in as short as 20 min per marker) [18], with the potential to enable multi-staining in routine service without disrupting the current laboratory workflow.

\section{CPATH and Post-Analytical Phase of IHC}

State-of-the-art computerized image analysis systems automatically evaluate and quantify IHC stains, not only minimize the inherent subjectivity of manual analysis and largely reduce the workload of pathologists via highthroughput analysis but also provide information beyond that pathologists can recognize. These include the spatial relationship between different types of cells and sub-cellular compartments, accurate assessment of intra-lesional heterogeneity, and more [26]. In a digital image analysis study that incorporated hexagonal tiling to compute intra-tumoural heterogeneity indicators for ER, PR, and $\mathrm{Ki}-67$ expression in breast cancer, the PR and Ki-67 intra- tumoural heterogeneity indicators were prognostically more informative than the rates of their expression [27]. The authors of that study suggested that prognostic modelling, based entirely on computational image-based IHC biomarkers, is possible, and that intra-tumoural heterogeneity and immune response indicators outperform both conventional breast cancer IHC and clinicopathological variables [27]. An automated digital image analysis methodology of the Ki-67 labelling index in breast cancer was proven superior to eyeballing assessment [28] and could determine optimal Ki-67 thresholds that were prognostically relevant [29]. Detection algorithms to quantify ER and PR expression and HER2 IHC status were developed and reported a correlation of more than $95 \%$ between manual and algorithmic quantification [2, 30]. These results demonstrate not only the robustness of the methodology and that AI-aided detection of IHC markers can perform as accurate as humans but also provide additional information beyond what human eyeballing assessment of such markers can provide with the potential to refine the existing classification systems.

Automated image analysis is associated with its problems as it depends more on the quality of the image and minor artefacts such as tissue folds, scores, and unspecific colourations, with an uneven colour potentially reducing analytic accuracy. Nevertheless, the technology is improving, and further adjustment of the systems with generalization capability is expected to overcome such limitations [28, 31]. Several studies have also used digital image analysis systems for analysing HER2, ER, and PR in breast cancer [32-34], and these showed that IHC image analysis results are similar to those obtained via the eyeball scoring method by expert pathologists.

\section{CPATH and Prediction of IHC Marker Expression (Virtual IHC)}

It is currently recognized that the capacity of CPATH and AI can identify new image features that may lead to the discovery of previously unrecognized morphological and biological characteristics with clinical relevance that cannot be identified using visual assessment by pathologists. The fact that deep learning is capable of using such "hidden" features is promising as it can yield important diagnostic, prognostic, and predictive information not currently utilized. With the advancement in computer vision and deep learning methods, some recent studies have demonstrated the ability of these techniques to replace chemical-based assays for molecular detection. The prediction of molecular expression of biomarkers in tissues based on evaluating the tissue architecture in WSI of un- 
stained or in haematoxylin-eosin (H\&E)-stained sections (virtual IHC or morphological-based molecular profiling using ML $[2,3,35])$, thus producing AI-based biomarkers.

In a previous study, Couture et al. [36] applied deep learning models to H\&E-stained WSI to predict the ER status and reported a final accuracy of $84 \%$. In a more recent study utilizing over 20,000 digitized, publicly available $\mathrm{H} \& \mathrm{E}$-stained sections from 5,356 patients with breast cancer, Shamai et al. [3] reported a significant correlation between tissue histomorphology and the molecular expression of all 19 biomarkers assayed, including ER, PR, and HER2. Prediction of ER expression showed an accuracy of $92 \%$ in at least half of the patients, which was noninferior to traditional IHC. Their results suggest that morphological-based molecular profiling could be used as a general approach for mass-scale molecular profiling, allowing quick, accurate, and inexpensive methods for simultaneous profiling of multiple IHC markers [3]. Rawat et al. [2] trained the algorithm to learn the morphological differences among different tumours to develop fingerprints that enabled the determination of ER, PR, and HER2 status from WSI of H\&E-stained slides with high accuracy (ER AUC $=0.89$, PR AUC $=0.81$, and HER2 AUC $=0.79$ ). Sha et al. [37] also used a deep learning model on WSI of H\&E-stained slides and predicted PDL1 status (AUC $=0.85$ ). Naik et al. [38] trained deep learning networks as per the multiple instances learning paradigm using only WSI-level labels on H\&E-stained WSIs from a multi-country dataset of 3,474 patients, achieving an AUC of 0.92. It must be noted that the utilization of multiple instance learning is important because it negates the necessity for pixel-level annotations of WSIs, an extremely costly and time-consuming process that is required as per the classic supervised learning paradigm. Anand et al. [39] trained a deep learning-based pipeline to determine the HER2 overexpression status of H\&E-stained WSIs in only 26 cases (11 HER2+ and 15 HER2-). The pipeline was tested on 26 (8 HER2+ and 18 HER2-) held-out cases from the same dataset and 45 TCGA-BRCA cohort cases (23 HER2+ and 22 HER2-), achieving AUCs of 0.82 and 0.76 , respectively.

\section{Al and IHC Quality Assurance}

The development and adoption of WSI in routine practice facilitated the adoption of computer vision and AI-based tools. The application of AI-based quality assurance and QC tools in clinical settings can have a substan- tial effect on IHC quality. AI can be leveraged to evaluate the staining characteristics of the tissue and combine findings with the predicted expression of specific markers, thereby confirming the results and identifying false staining patterns. With an increasing number of IHC marker assessments (mIHC), the QC and QA become more complex, and the use of AI algorithms and tools will be more relevant and needed. AI-based tools can also ensure a robust laboratory quality management process and provide a quality check on the pathologic diagnosis that may reduce errors.

It is important that the results of IHC generated by AIbased tools, whether image analysis of IHC-stained slides or predictions from H\&E-stained WSI, should be adequately validated and regulated, with appropriate governance oversight. Monitoring of diagnostic AI-based IHC assessment would include certification of the diagnostic laboratory, tools and personnel involved in the diagnostic interpretation of the results, assay performance assessment, proficiency testing, and adherence to specific practice guidelines. Regulatory bodies such as the Food and Drug Administration (FDA) and NICE regulate in vitro diagnostic tests including AI-based IHC tools as a part of medical practice. Therefore, strict measures involving not only the AI-based tool performance but also the local laboratory and specimen factors such as tissue and staining variables and scanners must be considered to obtain higher performing AI systems for interpretation of IHC. Commercial and academic entities will need to collaborate to bring the best-performing approaches over the regulatory finish line.

\section{Challenges to Al Application in Breast Pathology and Immunohistochemistry}

With the advancement of CPATH, several challenges remain to be solved including the low penetration rate of DP in routine practice, with a subsequent barrier to integrating AI-based tools into clinical workflows being the cost of such tools [16]. Research in the field of automated pathology diagnosis is rather demanding and requires a multitude of skills from different disciplines including computer scientists, pathologists, and others. However, developing and expanding this highly interdisciplinary field require researchers with experience in experimental studies as well as knowledge of diagnostic interpretation. Assuming that the AI-based tools prove efficient and reliable, a potentially challenging question remains: Will pathologists actually adopt these tools for IHC testing in 
routine practice? The most compelling argument for the adoption of such new technology would be an improvement in diagnostic accuracy and workflow efficiency. Automated tissue processing and staining tools have already made pathology work more efficient, and automatic IHC tools, either imaging analysis or AI-based virtual IHC tools, are likely to improve the performance. Evidence in the literature suggests that CPATH and AI-based quantitation of IHC biomarkers have significant benefits over manual scoring, such as increased objectivity, accuracy, reproducibility, and throughput and is likely to represent a milestone of digital health. When such advanced "nextgeneration" IHC is employed in pathology, it will help discover new perspectives in human biology.

\section{WSI Scanner Caused Domain Shift}

One problem that needs to be resolved before AI-powered pipelines can be readily utilized in real-world conditions is the variance originating from the devices that are used for digitizing the microscope slides, namely, the WSI scanners. Although human performance has been matched by deep learning models in histopathology given large amounts of properly annotated high-quality data, these models generalize poorly in real-laboratory conditions due to variance introduced by sample preparation, staining, and image digitization, a situation known as domain shift. It has been demonstrated that a major part of the domain shift is caused by the WSI scanners and is independent of sample preparation and staining kits [40]. Therefore, deep learning models trained over images originating from a single WSI scanner are expected to generalize poorly on images originating from another. Initiatives such as the MICCAI MIDOG 2021 international competition (REF: https://imi.thi.de/midog/) intend to provide the data and incentive to the scientific community to come up with solutions mitigating this effect. The challenge is focused on the task of mitosis detec- tion, which is directly related to tumour proliferation and as such highly relevant for driving the therapeutic strategy.

\section{Conclusions}

The widespread use of WSI technology for the primary diagnosis of breast pathology will enable the adoption of AI-based tools. AI applications in the field of breast pathology are increasing, including their use in diagnosis and prognosis. AI can improve IHC accuracy and provide information beyond "eyeball" visual assessment, with the potential to be an alternative to expensive multi-gene assays. Integration of AI into diagnostic pathology will help advance precision oncology and promote personalized care plans for each patient.

\section{Conflict of Interest Statement}

E.A.R. and P.H.T. are the guest editors of this special issue. K.V. has no conflicts of interest to declare.

\section{Funding Sources}

E.A.R. is part of the PathLAKE digital pathology consortium. These new Centres are supported by a $£ 50$ million investment from the Data to Early Diagnosis and Precision Medicine strand of the government's Industrial Strategy Challenge Fund, managed and delivered by UK Research and Innovation (UKRI).

\section{Author Contributions}

E.A.R. drafted the manuscript, reviewed and updated the various version of the manuscript, and approved the final version. P.H.T. reviewed and improved the manuscript, provided the image, and approved the final version. K.V. reviewed and improved the manuscript and approved the final version.

\section{References}

1 Abels E, Pantanowitz L, Aeffner F, Zarella MD, van der Laak J, Bui MM, et al. Computational pathology definitions, best practices, and recommendations for regulatory guidance: a white paper from the Digital Pathology Association. J Pathol. 2019;249:286-94.

2 Rawat RR, Ortega I, Roy P, Sha F, Shibata D, Ruderman D, et al. Deep learned tissue "fingerprints" classify breast cancers by ER/PR/ Her2 status from H\&E images. Sci Rep. 2020; 10:7275.
3 Shamai G, Binenbaum Y, Slossberg R, Duek I, Gil Z, Kimmel R. Artificial intelligence algorithms to assess hormonal status from tissue microarrays in patients with breast cancer. JAMA Netw Open. 2019;2:e197700.

4 Wang X, Zou C, Zhang Y, Li X, Wang C, Ke $F$, et al. Prediction of BRCA gene mutation in breast cancer based on deep learning and histopathology images. Front Genet. 2021;12: 661109.
5 Klimov S, Miligy IM, Gertych A, Jiang Y, Toss MS, Rida P, et al. A whole slide image-based machine learning approach to predict ductal carcinoma in situ (DCIS) recurrence risk. Breast Cancer Res. 2019;21:83.

6 Rakha EA, Toss M, Shiino S, Gamble P, Jaroensri R, Mermel CH, et al. Current and future applications of artificial intelligence in pathology: a clinical perspective. J Clin Pathol. 2021;74:409-14. 
7 Ibrahim A, Gamble P, Jaroensri R, Abdelsamea MM, Mermel CH, Chen PC, et al. Artificial intelligence in digital breast pathology: techniques and applications. Breast. 2020;49: 267-73.

8 Elsharawy KA, Gerds TA, Rakha EA, Dalton LW. Artificial intelligence grading of breast cancer: a promising method to refine prognostic classification for management precision. Histopathology. 2021;79(2):187-99.

9 Acs B, Rantalainen M, Hartman J. Artificial intelligence as the next step towards precision pathology. J Intern Med. 2020;288:62-81.

10 Tizhoosh HR, Pantanowitz L. Artificial intelligence and digital pathology: challenges and opportunities. J Pathol Inform. 2018;9:38.

11 Cui M, Zhang DY. Artificial intelligence and computational pathology. Lab Invest. 2021; 101(4):412-22.

12 Jiang Y, Yang M, Wang S, Li X, Sun Y. Emerging role of deep learning-based artificial intelligence in tumor pathology. Cancer Commun. 2020;40:154-66.

13 Dias R, Torkamani A. Artificial intelligence in clinical and genomic diagnostics. Genome Med. 2019;11:70.

14 Rakha EA, Pinder SE, Bartlett JM, Ibrahim M, Starczynski J, Carder PJ, et al. Updated UK recommendations for HER2 assessment in breast cancer. J Clin Pathol. 2015;68:93-9.

15 Hammond ME, Hayes DF, Wolff AC, Mangu $\mathrm{PB}$, Temin S. American society of clinical oncology/college of american pathologists guideline recommendations for immunohistochemical testing of estrogen and progesterone receptors in breast cancer. J Oncol Pract. 2010;6:195-7.

16 Cornish TC. Clinical application of image analysis in pathology. Adv Anat Pathol. 2020; 27:227-35.

17 Saeed AF, Wang R, Ling S, Wang S. Antibody engineering for pursuing a healthier future. Front Microbiol. 2017;8:495.

18 Cappi G, Dupouy DG, Comino MA, Ciftlik AT. Ultra-fast and automated immunohistofluorescent multistaining using a microfluidic tissue processor. Sci Rep. 2019;9:4489.

19 Morrison LE, Lefever MR, Behman LJ, Leibold T, Roberts EA, Horchner UB, et al. Brightfield multiplex immunohistochemistry with multispectral imaging. Lab Invest. 2020; 100:1124-36.

20 Wharton KA Jr, Wood D, Manesse M, Maclean KH, Leiss F, Zuraw A. Tissue multiplex analyte detection in anatomic pathology: pathways to clinical implementation. Front Mol Biosci. 2021;8:672531.
21 Tien TZ, Lee J, Lim JCT, Chen XY, Thike AA, Tan PH, et al. Delineating the breast cancer immune microenvironment in the era of multiplex immunohistochemistry/immunofluorescence. Histopathology. 2021;79:139-59.

22 Van Herck Y, Antoranz A, Andhari MD, Milli G, Bechter O, De Smet F, et al. Multiplexed immunohistochemistry and digital pathology as the foundation for next-generation pathology in melanoma: methodological comparison and future clinical applications. Front Oncol. 2021;11:636681.

23 Fassler DJ, Abousamra S, Gupta R, Chen C, Zhao M, Paredes D, et al. Deep learning-based image analysis methods for brightfield-acquired multiplex immunohistochemistry images. Diagn Pathol. 2020;15:100.

24 Day WA, Lefever MR, Ochs RL, Pedata A, Behman LJ, Ashworth-Sharpe J, et al. Covalently deposited dyes: a new chromogen paradigm that facilitates analysis of multiple biomarkers in situ. Lab Invest. 2017;97:104-13.

25 Black S, Phillips D, Hickey JW, Kennedy-Darling J, Venkataraaman VG, Samusik N, et al. CODEX multiplexed tissue imaging with DNA-conjugated antibodies. Nat Protoc. 2021;16:3802-35.

26 Casiraghi E, Huber V, Frasca M, Cossa M, Tozzi M, Rivoltini L, et al. A novel computational method for automatic segmentation, quantification and comparative analysis of immunohistochemically labeled tissue sections. BMC Bioinformatics. 2018;19:357.

27 Zilenaite D, Rasmusson A, Augulis R, Besusparis J, Laurinaviciene A, Plancoulaine B, et al. Independent prognostic value of intratumoral heterogeneity and immune response features by automated digital immunohistochemistry analysis in early hormone receptorpositive breast carcinoma. Front Oncol. 2020; 10:950.

28 Laurinavicius A, Plancoulaine B, Laurinaviciene A, Herlin P, Meskauskas R, Baltrusaityte I, et al. A methodology to ensure and improve accuracy of Ki67 labelling index estimation by automated digital image analysis in breast cancer tissue. Breast Cancer Res. 2014; 16:R35.

29 Tay TKY, Thike AA, Pathmanathan N, JaraLazaro AR, Iqbal J, Sng ASH, et al. Using computer assisted image analysis to determine the optimal Ki67 threshold for predicting outcome of invasive breast cancer. Oncotarget. 2018;9:11619-30.
30 Skaland I, Ovestad I, Janssen EA, Klos J, Kjellevold KH, Baak JP. Comparing subjective and digital image analysis HER2/neu expression scores with conventional and modified FISH scores in breast cancer. J Clin Pathol. 2008;61:68-71.

31 Barricelli BR, Casiraghi E, Gliozzo J, Huber V, Leone BE, Rizzi A, et al. ki67 nuclei detection and ki67-index estimation: a novel automatic approach based on human vision modeling. BMC Bioinformatics. 2019;20:733.

32 Khameneh FD, Razavi S, Kamasak M. Automated segmentation of cell membranes to evaluate HER2 status in whole slide images using a modified deep learning network. Comput Biol Med. 2019;110:164-74.

33 Li AC, Zhao J, Zhao C, Ma Z, Hartage R, Zhang Y, et al. Quantitative digital imaging analysis of HER2 immunohistochemistry predicts the response to anti-HER2 neoadjuvant chemotherapy in HER2-positive breast carcinoma. Breast Cancer Res Treat. 2020; 180:321-9.

34 Qaiser T, Rajpoot NM. Learning where to see: a novel attention model for automated immunohistochemical scoring. IEEE Trans Med Imaging. 2019;38:2620-31.

35 Lancellotti C, Cancian P, Savevski V, Kotha SRR, Fraggetta F, Graziano P, et al. Artificial intelligence \& tissue biomarkers: advantages, risks and perspectives for pathology. Cells. 2021:10:787.

36 Couture HD, Williams LA, Geradts J, Nyante SJ, Butler EN, Marron JS, et al. Image analysis with deep learning to predict breast cancer grade, ER status, histologic subtype, and intrinsic subtype. NPJ Breast Cancer. 2018;4:30.

37 Sha L, Osinski BL, Ho IY, Tan TL, Willis C, Weiss H, et al. Multi-field-of-view deep learning model predicts nonsmall cell lung cancer programmed death-ligand 1 status from whole-slide hematoxylin and eosin images. J Pathol Inform. 2019;10:24.

38 Naik N, Madani A, Esteva A, Keskar NS, Press MF, Ruderman D, et al. Deep learning-enabled breast cancer hormonal receptor status determination from base-level H\&E stains. Nat Commun. 2020;11:5727.

39 Anand D, Kurian NC, Dhage S, Kumar N, Rane S, Gann PH, et al. Deep learning to estimate human epidermal growth factor receptor 2 status from hematoxylin and eosinstained breast tissue images. J Pathol Inform. 2020;11:19.

40 Aubreville M, Bertram C, Veta M, Klopfleisch R, Stathonikos N, Breininger K, et al. Quantifying the scanner-induced domain gap in $\mathrm{mi}$ tosis detection. arXiv [preprint]. 2021. arxiv. org. arXiv:2103.16515 [cs.CV]. 